\title{
Molecular Theory of Rubber Elasticity
}

\author{
Paul J. FLORY \\ Department of Chemistry, Stanford University, \\ Stanford, California 94305, U.S.A.
}

(Received August 20, 1984)

\begin{abstract}
Rubber elasticity, identified as the capacity to sustain very large deformations followed by complete recovery, is exhibited exclusively by polymeric substances consisting predominantly of long molecular chains. Moreover, it is manifested under suitable conditions by virtually all polymers so constituted. The molecular theory of rubber elasticity rests on the premise, now fully validated by experiments, that alterations of the configurations of the chains comprising the network account for the elastic free energy and for the stress arising from deformation. Early theories of rubber elasticity were propounded on the assumption that displacements of the junctions are affine in the macroscopic strain. James and Guth, avoiding this assumption, treated a phantom network consisting of Gaussian chains having otherwise no material properties. They showed (i) that the mean positions of the junctions in this hypothetical network are affine in the strain, and (ii) that fluctuations about these positions are invariant under strain. The corollary that the instantaneous distribution of the chain vectors cannot be affine in the strain escaped notice for many years. The copious interpenetration of chains that characterizes polymer networks should be expected to restrain the fluctuations of junctions embedded therein, but not to suppress them altogether. Moreover, the restraints on fluctuations should depend on the state of strain. Departures from phantom behavior consequently occur to a degree that depends on the strain. Formulation of a self-consistent theory based on this idea required recognition of the non-affine connection between the chain vector distribution function and the macroscopic strain in a real network, which may partake of characteristics of a phantom network in some degree. Postulation of domains of constraint affecting the equilibrium distribution of fluctuations of network junctions from their mean positions led to a theory that accounts for the observed relationship of stress to strain virtually throughout the ranges accessible to measurement. The theory establishes connections between network structure and elastic properties. All essential parameters are determined by the connectivity of the network, the number and functionality of its junctions, and inherent characteristics of the molecular chains comprising the network.
\end{abstract}

KEY WORDS Rubber Elasticity / Elasticity Theory / Elastic Free Energy / Stress-Strain Relations / Network Structure / Entanglements / Junction Fluctuations / Non-Affine Networks /

The ability to sustain high deformations followed by full recovery upon removal of the stress is a property manifested under suitable conditions by virtually all polymeric substances consisting of long molecular chains. Moreover, it is exhibited exclusively by materials so constituted. This property assumes importance beyond the narrow limits of the term "rubber elasticity" by which it is commonly designated. It is operative in the swelling of polymeric networks and in the deformation of substances not generally included in the category of elastomers, e.g., in the deformation of semicrystal- line polymers and in the viscoelastic behavior of linear polymers under flow in the liquid or amorphous state. Rubber elasticity is essential to the functions of elastic proteins and muscle. The theory of rubber elasticity is centrally important to much of polymer science.

The basic premise of the molecular theory of rubber elasticity asserts that the stress in a typical strained elastomer originates within the molecular chains of the structure, typically a covalent network; contributions from interactions between the chains are negligible. This premise finds direct sup- 
port in elasticity measurements on polymeric networks. The temperature coefficient of the stress at fixed strain and its constancy with dilution are especially significant in this connection. ${ }^{1-4}$

Even more pervasive confirmation is provided by experiments showing the configurations of polymer chains to be unperturbed by their neighbors in amorphous polymers. Neutron scattering has been particularly decisive in demonstrating the absence of appreciable perturbations in the configurations of polymer chains when interspersed with other randomly configured polymer molecules of the same kind. 5 The (free) energy of interaction between neighboring chains must therefore be sensibly independent of their configurations. It follows that the intermolecular energy should not be significantly affected by the changes in configurations of the chains of a network induced by deformation. ${ }^{7}$ The stored elastic free energy, which is central to the theory of rubber elasticity, therefore comprises the sum of contributions of the individual network chains. Other contributions, such as often have been postulated to arise from interchain interactions, may be ignored according to the stated premise and the compelling evidence in its support.

The principal task of theory is to establish the relationship between the macroscopic strain and the distortion of the distribution of configurations of the network chains. ${ }^{7,8}$ It was assumed originally that the locations of the network junctions may be considered to be affine in the macroscopic strain, from which it followed that the distribution of endto-end vectors of the chains, i.e., the chain vectors, should likewise be affine in the strain. ${ }^{9-14}$ The theory of James and Guth ${ }^{15}$ appeared at first to corroborate this conjecture, inasmuch as it showed the mean locations of junctions in a "phantom network" (see below) of Gaussian chains to be affine in the strain. Nearly thirty years elapsed before the important distinction between the distribution of mean chain vectors and their instantaneous (or time averaged) distribution was recognized. ${ }^{7}$ Modern theory of rubber elasticity is an outgrowth of the recognition that the actual distribution of chain vectors in a network of Gaussian chains is, in general, non-affine in the strain.

\section{THE ISOLATED CHAIN}

The chains in elastomeric networks typically con- sist of $100-1000$ skeletal bonds. For free chains of this length, the function describing the distribution of end-to-end chain vector $\boldsymbol{r}$ is Gaussian in good approximation $;^{16}$ i.e., the distribution is well represented by

$$
W(\boldsymbol{r})=\left(3 / 2 \pi\left\langle r^{2}\right\rangle_{0}\right)^{3 / 2} \exp \left[-\left(3 / 2\left\langle r^{2}\right\rangle_{0}\right) r^{2}\right]
$$

where $\left\langle r^{2}\right\rangle_{0}$ is the mean-square magnitude of $\boldsymbol{r}$ for the free chain averaged over all configurations. It follows that the free energy of the chain is given as a function of its displacement length $r \equiv|\boldsymbol{r}|$ by

$$
\begin{aligned}
A(\boldsymbol{r}) & =\mathrm{const}-k T \ln W(\boldsymbol{r}) \\
& =A^{0}(T)+\left(3 k T / 2\left\langle r^{2}\right\rangle_{0}\right) r^{2}
\end{aligned}
$$

The magnitude of the average retractive force exerted by the chain at fixed $r$, obtained by differentiation of eq 2 , is

$$
\bar{f}=3 k T\left\langle r^{2}\right\rangle_{0}^{-1} r
$$

It is directed along the chain vector. Proportionality between the average force and the displacement length of the chain follows directly from eq 1 , as is obvious. Conversely, primary assertion of eq 3 would lead to eq 1 .

According to the premise enunciated above, the elastic properties of a network of Gaussian chains must follow from these relationships.

\section{NETWORK STRUCTURE AND TOPOLOGY}

A polymer network may be characterized by the number $\mu_{\mathrm{J}}$ of its junctions, their functionality $\phi$ (or average functionality $\bar{\phi}$ ), and by the number $v_{\text {ends }}$ of ends of chains. ${ }^{7}$ The number of chains in the network, including those with only one end attached, is

$$
v=(1 / 2)\left(\mu_{\mathrm{J}} \phi+v_{\text {ends }}\right)
$$

The effective number $v_{\mathrm{e}}$ of chains is less than $v$ owing to the imperfections due to free chain ends. For a perfect network for which $v_{\text {ends }}=0$,

$$
v_{\mathrm{e}}=v=\mu_{\mathrm{J}} \phi / 2
$$

A quantity that characterizes the network with greater generality, regardless of the nature of its imperfections, is the cycle rank $\xi$, or number of independent circuits it contains. ${ }^{7,17}$ It may be defined alternatively as the minimum number of scis- 


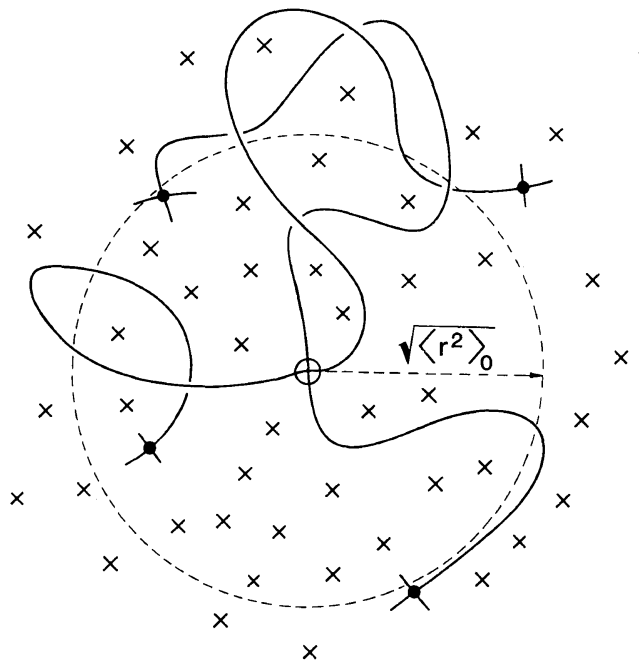

Figure 1. Spatial neighbor junctions $\times$ surrounding a given junction and its four topological neighbors in a tetrafunctional network.

sions required to reduce the network to a "spanning tree," i.e., a unified structure comprising all of the chains and containing no closed circuits or loops. This quantity will be used in due course to characterize the elastic response of the network. It suffices to observe that in a perfect network $\xi$ is given by the difference between the number of chains and the number of junctions of functionality $\phi \geq 3$; i.e.,

$$
\xi=v-\mu_{\mathrm{J}}=v(1-2 / \phi)
$$

See eq 5.

A prominent and important feature that is characteristic of polymeric networks is the copious interpenetration of chains and junctions. ${ }^{7,18}$ The region of space pervaded by a given chain is shared with many other chains and junctions. The domain roughly demarcated by the junctions that are topologically first neighbors of a given junction is occupied by many other junctions. This is illustrated in Figure 1 for a tetrafunctional network.

The average number $\Gamma$ of junctions within the region of radius $\left\langle r^{2}\right\rangle_{0}^{1 / 2}$ offers a quantitative measure of the degree of interpenetration. It is given by

$$
\Gamma=(4 \pi / 3)\left\langle r^{2}\right\rangle_{0}^{3 / 2}\left(\mu_{\mathrm{J}} / V^{0}\right)
$$

where $V^{0}$ is the volume of the network in its state of reference (see below). Since $\left\langle r^{2}\right\rangle_{0}$ increases linearly with the length of a chain, it must increase linearly with $\mu_{\mathrm{J}}^{-1}$. Hence, $\Gamma$ is inversely proportional to the square-root of the degree of interlinking. For typical elastomeric networks, $\Gamma$ is in the range 25-100. Clearly, the chains and junctions are profusely interspersed. The first tier of topological neighbors is located well beyond the nearest spatial neighbors; see Figure 1. It is to be noted also that the shortest topological pathway from a given junction to one of its nearest neighbors in space may span many chains.

\section{AFFINE NETWORKS}

The high degree of interpenetration in elastomeric networks and the fact that each junction is located in an environment dominated by chains and junctions whose structural relation to the junction considered is remote lends credence to the assumption, universally adopted in the earliest theories of rubber elasticity, ${ }^{9-14}$ that the positions of the junctions are approximately affine in the macroscopic strain. ${ }^{13}$ It follows at once from the premise introduced above that the elastic free energy of the network is the sum of expressions like eq 2 for each chain of the network. Required is the sum $\sum_{i=1}^{v} r_{i}^{2}$ over all chains. According to the assumption that the transformation of chain vectors is affine in the displacement gradient tensor $\lambda$ that defines the macroscopic strain, this sum is just $v\left\langle r^{2}\right\rangle_{0}\left(\lambda_{x}^{2}+\right.$ $\lambda_{y}^{2}+\lambda_{z}^{2}$ ), where $\lambda_{x}, \lambda_{y}, \lambda_{z}$ are the principal extension ratios measured relative to the dimensions of the specimen when isotropic and at the volume $V^{0}$ such that the mean-square magnitude of the chain vectors matches the value $\left\langle r^{2}\right\rangle_{0}$ for unperturbed chains. Adding the term $-\mu_{\mathrm{J}} k T \ln V$ for the dispersion of the junctions over the prevailing volume $V$ and expressing the free energy relative to the state of reference in which $\lambda_{x}=\lambda_{y}=\lambda_{z}=1$ and $V=$ $V^{0}$, one obtains ${ }^{14,19}$

$$
\begin{aligned}
\Delta A_{\text {aff }}= & (v / 2) k T\left(\lambda_{x}^{2}+\lambda_{y}^{2}+\lambda_{z}^{2}-3\right) \\
& -\mu_{\mathrm{J}} k T \ln \left(V / V^{0}\right)
\end{aligned}
$$

The stress is obtained as a function of strain by differentiation of eq 8 . For uniaxial elongation parallel to the $X$-axis, $\lambda_{x}=\lambda=L / L^{0}$ and $\lambda_{y}=\lambda_{z}=$ $\left(V / V_{0} \lambda\right)^{1 / 2}$, and the force of retraction for the affine network is 


$$
\begin{aligned}
f_{\text {aff }} & =\left(\partial \Delta A_{\mathrm{el}} / \partial L\right)_{\mathrm{T}, \mathrm{v}}=\left(\partial \Delta A_{\mathrm{el}} / \partial \lambda\right)_{\mathrm{T}, \mathrm{v}} / L^{0} \\
& =\left(v k T / L^{0}\right)\left(\lambda-V / V^{0} \lambda^{2}\right) \\
& =\left(v k T / L_{\mathrm{i}, \mathrm{v}}\right)\left(V / V^{0}\right)^{2 / 3}\left(\alpha-\alpha^{-2}\right)
\end{aligned}
$$

where $\alpha=L / L_{\mathrm{i}, \mathrm{V}}=\lambda\left(V / V^{0}\right)^{-1 / 3}$ is the extension ratio relative to the length $L_{\mathrm{i}, \mathrm{V}}=L^{0}\left(V / V^{0}\right)^{1 / 3}$ of the unstretched (isotropic) specimen at the volume $V$ prevailing in the elongated state. Equations 9 and 10 are traditionally identified as alternative stressstrain relations for Gaussian networks.

\section{PHANTOM NETWORKS}

The theory of James and Guth, ${ }^{15}$ which appeared in 1947, is a landmark in the evolution of rubber elasticity theory. It addresses networks of Gaussian chains whose only action is to deliver contractile forces (proportional to their displacement lengths $r$ ) at the junctions to which they are attached. The chains have no other material properties; they may pass through one another freely and they are not subject to the volume exclusion requirements of real molecular systems. Being free of constraints by neighboring chains, the junctions of the "phantom network" thus described undergo displacements that are affected only by their connections to the network and not at all by their immediate surroundings.

Without prior assumptions concerning the disposition of the junctions in a Gaussian phantom network, James and Guth ${ }^{15}$ showed (i) that their mean positions in this hypothetical network are affine in the strain, (ii) that their fluctuations about these mean positions are Gaussian, and (iii) that these fluctuations should be independent of the strain. The fluctuations of the junctions are substantial. The mean-squared magnitude of the fluctuations in the chain vectors caused by them is given by $^{20,21}$

$$
\left\langle(\Delta r)^{2}\right\rangle=(2 / \phi)\left\langle r^{2}\right\rangle_{0}
$$

The corresponding measure of the dispersion in the magnitudes of the mean vectors is ${ }^{7}$

$$
\left\langle\bar{r}^{2}\right\rangle=(1-2 / \phi)\left\langle r^{2}\right\rangle_{0}
$$

Thus, for a tetrafunctional phantom network, the fluctuations account for half of $\left\langle r^{2}\right\rangle_{0}$ for the free chain.

It follows directly from the James and Guth deductions (i) and (iii) above that the instantaneous positions must be non-affine in the strain: the distribution of junctions is the convolution of their mean positions, which are affine in the strain, with their fluctuations which are invariant with strain. The distribution of chain vectors in the phantom network, although a function of the strain, is not therefore affine in the strain. We return later to the implications of this long-overlooked corollary of the James and Guth theory.

This theory ${ }^{15}$. leads to an elastic free energy of the same form as the first term in eq 8 , but with a smaller coefficient. For a tetrafunctional network $v / 2$ should be replaced by $v / 4$ in the adaptation of that equation to a phantom network. Additionally, the second term of eq 8 disappears. As was shown subsequently, ${ }^{7}$ the elastic free energy for a phantom network with junctions of any functionality is given with complete generality by

$$
\Delta A_{\mathrm{ph}}=(\xi / 2) k T\left(I_{1}-3\right)
$$

where $\xi$ is the cycle rank (see above) and $I_{1}$ is the first strain invariant defined by

$$
I_{1}=\lambda_{x}^{2}+\lambda_{y}^{2}+\lambda_{z}^{2}
$$

The form of the dependence of the force of retraction on strain under uniaxial deformation is the same as given by eq 9 or 10 . The number $v$ of chains is replaced by $\xi$. Hence, the retractive force is

$$
\begin{aligned}
f_{\mathrm{ph}} & =\left(\xi k T / L_{\mathrm{i}, \mathrm{V}}\right)\left(V / V^{0}\right)^{2 / 3}\left(\alpha-\alpha^{-2}\right) \\
& =\left(\xi k T / L^{0}\right)\left(V / V^{0}\right)^{1 / 3}\left(\alpha-\alpha^{-2}\right)
\end{aligned}
$$

For a perfect tetrafunctional network $\xi=v / 2$, as follows from eq 6 . Hence, the predicted retractive force in this case is half that for the affine network. This difference reflects the fact that only the mean vectors $\boldsymbol{r}$ are altered by the strain; the fluctuations, which in a tetrafunctional network account for half of $\left\langle r^{2}\right\rangle_{0}$, are unaffected by strain.

\section{COMPARISONS OF AFFINE AND PHANTOM NETWORK THEORIES WITH EXPERIMENTS}

It follows from eq 10 and likewise from eq 15 that

$$
\begin{aligned}
{[\partial \ln (f / T) / \partial T]_{\mathrm{L}, \mathrm{V}} } & =-(2 / 3) \mathrm{d} \ln V^{0} / \mathrm{d} T \\
& =-\mathrm{d} \ln \left\langle r^{2}\right\rangle_{0} / \mathrm{d} T
\end{aligned}
$$


Values of $\mathrm{d} \ln \left\langle r^{2}\right\rangle_{0} / \mathrm{d} T$ determined from stresstemperature coefficients using this relationship are not appreciably affected by swelling of the network with a diluent. ${ }^{1-4}$ They are in agreement with results of measurements conducted on dilute solutions of the linear polymer. ${ }^{3,22}$ These findings lend assurance that the primary premise of the molecular theories of rubber elasticity is valid.

The experimental relationship of stress to strain is strikingly at variance with the traditional theories discussed above. Gee ${ }^{23}$ showed in 1946 that the slope of the tension-elongation curve observed for natural rubber diminishes more rapidly with elongation and with swelling than is predicted by eq 10 , and hence also by eq 15 . Similar departures from theory were found for other elastomers. ${ }^{8,14,23}$ The disparity between the factors of proportionality in eq 10 and 15 representing theories for affine and phantom networks, respectively, was overshadowed by the failure of both theories to account for the relationship of stress to strain.

This circumstance led to widespread adoption of the Mooney-Rivlin relation obtained by arbitrarily appending a term proportional to the second strain invariant, $I_{2}=\lambda_{x}^{2} \lambda_{y}^{2}+\lambda_{y}^{2} \lambda_{z}^{2}+\lambda_{z}^{2} \lambda_{x}^{2}$, to the elastic free energy. The resulting relationship of the tension to elongation is

$$
f=2 C_{1}\left(\alpha-\alpha^{-2}\right)+2 C_{2}\left(1-\alpha^{-3}\right)
$$

or

$$
f /\left(\alpha-\alpha^{-2}\right)=2 C_{1}+2 C_{2} / \alpha
$$

where $C_{1}$ and $C_{2}$ are empirical constants for a given elastomer at a fixed temperature. Agreement with the observed tension-elongation relationship in simple extension is improved through use of eq 17 having the additional parameter $C_{2}$. It fails utterly in compression (or equibiaxial extension) and for biaxial strains generally. ${ }^{8}$ Even in simple elongation, departures from the linear relation prescribed by eq 17 are apparent.

With the main focus of attention on elastomers in uniaxial elongation, experiments indicated that the "correction term" in $C_{2}$ diminishes with dilation (swelling) and that it diminishes relative to $C_{1}$ with increase in the degree of interlinking. This was implicit in the work of Gee. ${ }^{23}$ The results of Allen, Kirkham, Padget and Price ${ }^{2}$ shown in part in Figure 2 are particularly revealing in this connection. Here the reduced nominal stress defined by
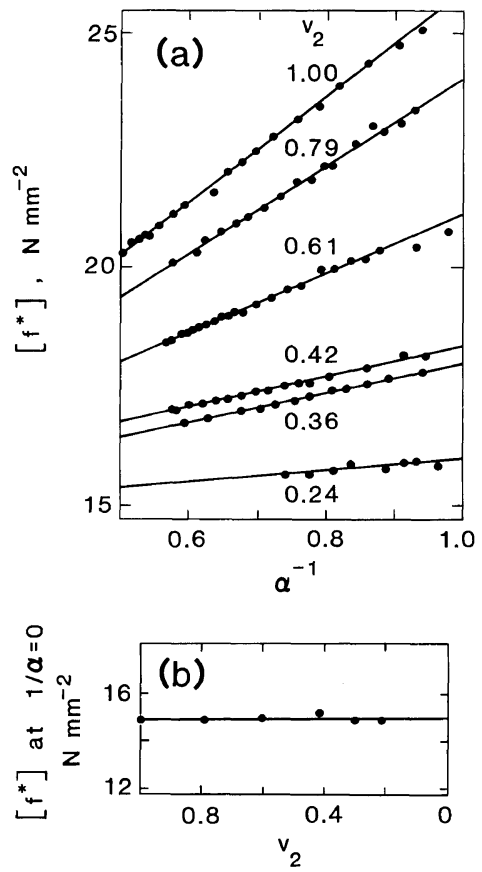

Figure 2. From ref 2.

$$
\left[f^{*}\right] \equiv\left(f / A^{0}\right) v_{\mathbf{p}}^{1 / 3}\left(\alpha-\alpha^{-2}\right)^{-1}
$$

where $A^{0}$ is the area of the initial cross section in the reference state, is plotted against $\alpha^{-1}$ in keeping with eq 17. The volume fraction $v_{\mathrm{p}}$ of rubber may be identified with $V^{0} / V$ in eq $15^{\prime}$, according to which [ $\left.f^{*}\right]$ should be a constant equal to $\xi k T / V^{0}$ for a given network. The slopes $\left(2 C_{2}\right)$ of the MooneyRivlin plots decrease with dilution, but the intercepts $\left(2 C_{1}\right)$ remain approximately the same. Other experiments reported by Allen et al..$^{2}$ showed that the intercept increases with degree of crosslinking. Thus, the intercept appears to be an invariant that characterizes a given network. Observations such as these suggested that $2 C_{1}$ may be identified with $\xi k T / V^{0}$ of eq $15^{\prime}$.

\section{FLUCTUATIONS IN REAL NETWORKS}

Inasmuch as the basic premise that the stored elastic free energy resides within the chains is fully validated, observed departures from the form of the stress-strain relationship prescribed by the theories cited above implicate the connections assumed, or deduced, between the macroscopic strain and the 


\section{P. J. FLORY}

distribution of chain vectors as the source of the discrepancy. The assumption that the latter are affine in the strain makes no allowance whatever for excursions of the junctions from their mean positions. Fluctuations of this nature are implicit in the molecular mobility that is essential to the high compliance exhibited by elastomers. It would be incorrect therefore to assume that they are suppressed altogether. On the other hand, the large, unimpeded fluctuations deduced for phantom networks may be curtailed severely by the profusion of chains in which each junction is embedded; see Figure $1 .^{24}$

Considerations such as these led Ronca and Allegra $^{25}$ and the author ${ }^{7}$ to suggest that real networks may behave in a manner between the two extremes. It was suggested further that a shift in proximity to these respective extremes might be expected with strain, phantom behavior being more closely approached with elongation or dilation..$^{7,25}$ Inasmuch as the factor of proportionality to the strain function is smaller according to phantom theory than for affine theory, the observed departures from these theories might thus be explained.

Exploitation of this conjecture requires full grasp of the implications of the non-affineness of the transformation of the distribution of chain vectors with strain. ${ }^{7}$ It is a necessary and sufficient condition for affine transformation that the neighborhood of junctions about a given junction be preserved, with distances between junctions altered in accordance with the displacement gradient $\lambda$. The environments of the junctions must theorefore change with deformation in a phantom (hence, non-affine) network, or, indeed, in any network in which the junctions undergo independent fluctuations. ${ }^{26,27}$ The magnitude of the fluctuations occurring in a phantom network being generally greater than the distance to the nearest spatial neighbors, drastic reshuffling of neighbors about a given junction may be required when the strain is large.

Extensive interpenetration of portions of the network that are topologically remote in structural relation to one another implies a maze of entanglements in which chains and junctions are inextricably intertwined. The mutual entanglement of chains and junctions confers a coherence on the real network not present in its phantom analog comprising chains that neither preempt space nor obstruct transection of one another. This is a feature of real networks that is of foremost importance. Occurrence of the rearrangements required by phantom network theory must obviously be difficult in a real network. ${ }^{26-28}$

The entanglements here referred to are not discrete in the sense that they engage a given chain with one of its neighbors in a unique relationship. Instead, they involve a given chain diffusely with the manifold of its neighbors. Contrary to the usual sketches of chain configurations, their trajectories do not oscillate back and forth as if guided by their time-averaged destinations. ${ }^{29}$ As follows from random-walk statistics in general, they are not selfcorrecting such that an excursion in a given direction presages correction by an opposing course. The instantaneous configuration of the chain seldom describes a path such as would wind it about a neighboring chain, thereby establishing an entanglement that could be equated to a cross-linkage. The diffuse entanglements prevalent in polymer networks allow extensive local rearrangements while, at the same time, precluding gross displacements of neighboring members of the network.

The number of configurations accessible to a network obviously is greatly reduced by the integrity of its permanent connections and by the further constraints due to entanglements. This reduction is inconsequential in the undeformed network formed by interlinking randomly configured, unperturbed chains. It is the average over configuration space for an ensemble of equivalently formed networks that is relevant to the treatment of equilibrium properties. The ensemble average is unaffected by interlinking of the chains, which occurs via a random process. Upon deforming the networks thus formed, constraints due to the physical integrity of the network, augmented by the effects of entanglements, contribute to the elastic free energy $\Delta A_{\mathrm{el}}$.

The network junctions are the members of the network most susceptible to the steric constraints imposed by the diffuse entanglements. Each of them marks the confluence of $\phi$ chains $(\phi \geq 3)$ that encumber displacement of the junction relative to its neighbors. Although constraints obviously impinge on the chains as well, the totality of all constraints may be treated, presumably in good approximation, as if they restrict displacements of the junctions exclusively. 


\section{THEORY OF NETWORKS WITH JUNCTIONS SUBJECT TO STRAIN-DEPENDENT CONSTRAINTS ${ }^{26,28}$}

The model adopted for the purpose of giving quantitative expression to the ideas above is shown in Figure 3. Point A represents the mean position of the chosen junction in the hypothetical phantom state of the network. The radius of the large dashed circle centered at $\mathrm{A}$ represents the root-mean-square fluctuation $\left\langle(\Delta R)^{2}\right\rangle_{\mathrm{ph}}^{1 / 2}$ about this position in the phantom state. The domain of constraints due to entanglements with surrounding real chains and to their steric requirements is represented by the smaller dashed circle centered at B and separated from A by $\bar{s}$. It may be considered to be located as if the constraints were suddenly imposed at an instant during which a random excursion of the junction about its mean position A carried it to point $B$. (The manner in which the network was actually formed is irrelevant.) After the constraints have been established, the mean position of the junction is at point $\mathrm{C}$ removed from $\mathrm{A}$ by $\Delta \bar{R}$. In other words, under the combined influences of its connections with the network (i.e., the phantom network forces) and of the constraints, the mean position of the junction in the unstrained, real network is at C. The instantaneous position of the junction happens to be at point $\mathrm{D}$, which is outside the domain of constraints by neighbors but inside the domain representing fluctuations of the phantom network. Both of the domain boundaries are diffuse rather than rigid; hence the junction may wander beyond either of them, although the probability of its doing so diminishes with the distances from their centers. For simplicity and without significant sacrifice of accuracy, we take the action of the domain of constraint to be a Gaussian function of the distance $\Delta s$ of the junction from $\mathrm{B}$, just as the action of the (phantom) network is a Gaussian function of $\Delta R$.

The principal parameter $\kappa$ that characterizes the constraints specifies the inverse ratio of the meansquare radii of the domains; i.e.,

$$
\kappa=\left\langle(\Delta R)^{2}\right\rangle_{\mathrm{ph}} /\left\langle(\Delta s)^{2}\right\rangle_{0}
$$

where $\left\langle(\Delta s)^{2}\right\rangle_{0}$ is the mean-square of the fluctuations about $B$ that would occur in the undeformed network if the junction would be subject only to the effects of its involvements with the surrounding chains, constraints imposed by its connections to the network being somehow suspended. Thus, $\kappa$ measures the severity of the entanglement constraints relative to those of the phantom network.

Since the network is formed through random molecular processes, the instantaneous distribution of junction positions, and hence of chain vectors, must be unaffected by formation of the network. It follows that the distribution of the centers of the domains of constraint about the mean phantom positions (A) must be identical, in the unstrained state, with the distribution of fluctuations $(\Delta R)$ in the phantom network.

Isotropy of the network in its state of rest implies that displacement of the centers of the domains of constraint should be affine under strain. The dimensions of these domains, unlike those representing the action of the phantom network, must undergo distortion under strain. In first approximation, ${ }^{26,28}$ they may be expected to become ellipsoidal according to the macroscopic deformation gradient tensor $\lambda$, i.e., the sphere represented in Figure 3 by the smaller dashed circle becomes an ellipsoid. Thus, if $\Delta x$ is the component of $\Delta s$ along one of the principal axes of $\lambda$, then on the assumption that the vectors $\Delta s$ are affine in $\lambda$

$$
\left\langle(\Delta x)^{2}\right\rangle_{\lambda} /\left\langle(\Delta x)^{2}\right\rangle_{0}=\lambda^{2}
$$

where $\lambda \equiv \lambda_{x}$. For $\lambda>1$, the domain of constraint is lengthened and the severity of the constraints is diminished in this direction.

Experimental results suggest a somewhat more rapid alteration of the constraints with strain than predicted by affine deformation of the domain. ${ }^{26,28,29}$ This observation may reflect structural inhomogeneities in the network. A higher approximation is offered by ${ }^{28}$

$$
\left\langle(\Delta x)^{2}\right\rangle_{\lambda} /\left\langle(\Delta x)^{2}\right\rangle_{0}=\lambda^{2}[1+\kappa \zeta(\lambda-1)]
$$

where $\zeta$ is an additional parameter. In the following development we neglect $\zeta$, although its effect on numerical calculations will be indicated.

The primary contribution to the elastic free energy from the connectivity of the network, i.e., the phantom network contribution $\Delta A_{\mathrm{ph}}$, is implicit in the displacement of the mean positions of the junctions in the phantom state. It is given by eq 13 . The contribution $\Delta A_{\mathrm{c}}$ from the steric constraints comprises two terms ${ }^{26,28}$ due, respectively, to (a) 


\section{P. J. FLORY}

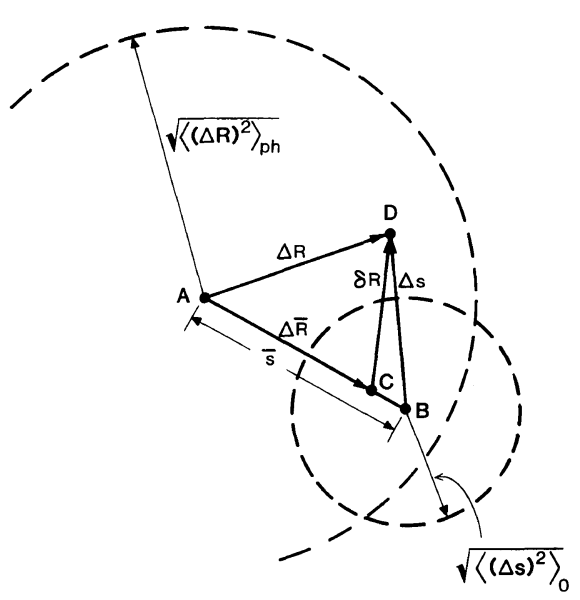

Figure 3. From ref 28.

alteration of the (instantaneous) distribution of the $\Delta \boldsymbol{R}$ (see Figure 3) from their values in the phantom network, and (b) alteration of the distribution of displacements $\Delta s$ of the junctions about the centers of their domains of constraint. The foregoing relations allow the required distributions to be formulated as functions of the strain. The free energies may then be obtained from the familiar configuration function

$$
\Omega=\prod_{i}\left(\omega_{i} \mu_{\mathrm{J}} / \mu_{\mathbf{J}, i}\right)^{\mu_{\mathbf{J}, i}}
$$

where $\mu_{\mathrm{J}, i}$ is the number of junctions at the location $\Delta \boldsymbol{R}_{i}$, or at $\Delta s_{i}$ relative to the center of the domain of constraint; $\omega_{i}$ is the a priori probability of the state thus specified, as given by the three-dimentional Gaussian probability distribution $W(\Delta \boldsymbol{R})$ or $W_{\mathrm{c}}(\Delta s)$, the latter being ellipsoidal under strain. The contributions (a) and (b) above follow from $-k T \ln \Omega$.

The total elastic free energy thus derived is just the sum

$$
\Delta A_{\mathrm{el}}=\Delta A_{\mathrm{ph}}+\Delta A_{\mathrm{c}}
$$

of the elastic free energy of the phantom network, $\Delta A_{\mathrm{ph}}$ given by eq 13 , and $\Delta A_{\mathrm{c}}$ for the combined contributions (a) and (b) above due to action of the constraints. According to the theory ${ }^{26,28}$ here outlined.

$$
\begin{aligned}
(k T)^{-1} \Delta A_{\mathrm{c}}= & \left(\mu_{\mathrm{J}} / 2\right) \sum_{t}\left\{\left(1+\lambda_{t}^{2} \kappa^{-1}\right) B_{t}\right. \\
& \left.-\ln \left[\left(B_{t}+1\right)\left(\lambda_{t}^{2} \kappa^{-1} B_{t}+1\right)\right]\right\}
\end{aligned}
$$

where

$$
B_{t}=\left(\lambda_{t}^{2}-1\right) /\left(\lambda_{t}^{2} \kappa^{-1}+1\right)^{2}
$$

and $t$ identifies the principal axis $x, y$ or $z$.

It will be apparent that $\Delta A_{\mathrm{c}}$ vanishes for $\kappa \rightarrow 0$. In the opposite limit where $\kappa^{-1} \rightarrow 0$,

$$
\Delta A_{\mathrm{c}}=\frac{1}{2} \mu_{\mathrm{J}} k T\left[I_{1}-3-2 \ln \left(V / V^{0}\right)\right]
$$

which, when substituted in eq 23 together with eq 13, followed by replacement of $\xi+\mu$, with $v$ according to eq 6 , yields $\Delta A_{\text {aff }}$ of eq $8 .{ }^{26}$ The present theory is therefore consistent with both affine and phantom theory at its respective limits.

The stress may be expressed similarly as the sum of contributions from the phantom network and from the entanglement constraints. In the case of simple elongation, for example, the tensile force is $^{26,28}$

$$
f=f_{\mathrm{ph}}+f_{\mathrm{c}}=f_{\mathrm{ph}}\left(1+f_{\mathrm{c}} / f_{\mathrm{ph}}\right)
$$

where $f_{\mathrm{ph}}$ is given by eq 15 or $15^{\prime}$ : The relative contribution from the entanglement constraints is

$$
f_{\mathrm{c}} / f_{\mathrm{ph}}=\left(\mu_{\mathrm{J}} / \xi\right)\left[\alpha K\left(\lambda_{1}^{2}\right)-\alpha^{-2} K\left(\lambda_{2}^{2}\right)\right]\left(\alpha-\alpha^{-2}\right)^{-1}
$$

where

$$
\lambda_{1}=\alpha\left(V / V^{0}\right)^{1 / 3} \text { and } \lambda_{2}=\alpha^{-1 / 2}\left(V / V^{0}\right)^{1 / 3},
$$

and

$$
\begin{aligned}
K\left(\lambda^{2}\right)= & B\left[\dot{B}(B+1)^{-1}\right. \\
& \left.+\kappa^{-1}\left(\lambda^{2} \dot{B}+B\right)\left(\kappa \lambda^{-2}+B\right)^{-1}\right]
\end{aligned}
$$

where

$$
\dot{B}=\partial B / \partial \lambda^{2} .
$$

In general, and for perfect networks in particular, $\mu_{\mathrm{J}} / \xi$ may be replaced by unity in eq 27 .

\section{COMPARISON OF THEORY WITH EXPERIMENTS}

Experimental results on elastomers in uniaxial strain are conveniently represented by plots of the reduced nominal stress $\left[f^{*}\right]$ (see eq 18) against $\alpha^{-1}$. Results of $\mathrm{Pak}^{30}$ covering an exceptionally wide range of extension ratio $\alpha$ are shown by the points in Figure $4 .^{29}$ The experiments were carried out on cross-linked poly(dimethylsiloxane), PDMS, without dilation, i.e., with $v_{2}=1$. Those in compression, for which $\alpha^{-1}>1$, were obtained ${ }^{30}$ by 


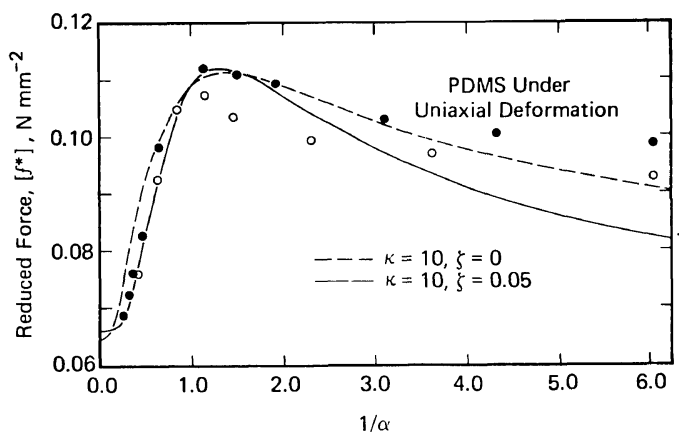

Figure 4. Results of $\mathrm{Pak}^{30}$ on PDMS in extension $(1 / \alpha<1)$ and compression $(1 / \alpha>1)$. From ref 29 .

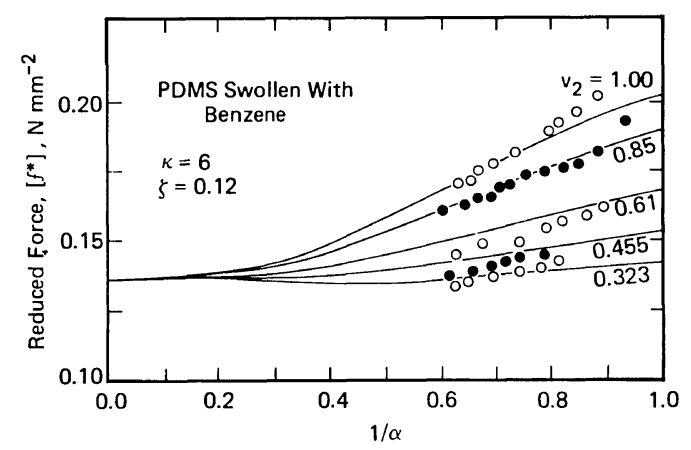

Figure 5. Results of Flory and Tatara. ${ }^{31}$ Effects of swelling on cross-linked PDMS. From ref 29.

measuring the inflation of a sheet as a function of pressure. Measurements in extension were conducted on strips from the same sample. The curves have been calculated according to the theory discussed above using the parameters indicated. Use of $\zeta=0.05$ instead of $\zeta=0$ improves the agreement with experiment in compression $(1 / \alpha>1)$ but at the expense of agreement in extension. The divergence between theory and experiment is small compared to the range covered: fourfold in extension and sixfold in compression.

The results of Allen et al. ${ }^{2}$ on the effects of swelling on the reduced force of networks of natural rubber (see Figure 2) are well represented by theory. ${ }^{29}$ Results of Tatara ${ }^{31}$ on PDMS networks swollen to the various degrees indicated by the volume fractions $v_{2}$ of polymer are compared in Figure 5 with calculations according to theory for the values of $\kappa$ and $\zeta$ indicated.

Results of Mark and Sullivan ${ }^{32}$ on tetrafunctional networks prepared by end-linking PDMS chains of different lengths, shown in Figure 6,

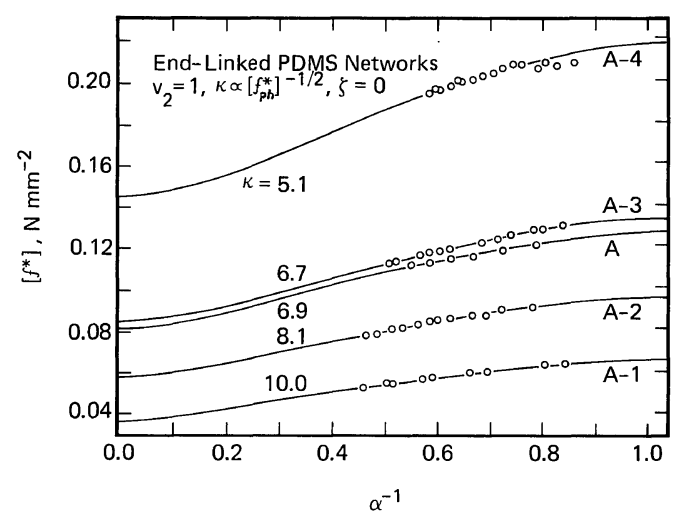

Figure 6. Results of Mark and Sullivan ${ }^{32}$ on endlinked PDMS. From Erman and Flory. ${ }^{29}$

demonstrate the effect of the degree of cross-linking. The curves have been calculated for values of $\kappa$ chosen to be inversely proportional to the squareroots of the degrees of cross-linking (i.e., $\kappa \propto \xi^{-1 / 2}$ ) on the hypothesis that the constraints should be proportional to the degree of interpenetration; ${ }^{29}$ see eq 7. All of the data for the several networks are well reproduced by the arbitrary choice of one of the $\kappa$ 's, the others being related thereto through the degrees of cross-linking.

Measurements of stress in biaxial extension are more definitive inasmuch as the strain is bivariate. The more complex array of data obtained from skilfully executing experiments ${ }^{33}$ on rubber in biaxial strain long resisted rational interpretation ${ }^{8}$ on a molecular basis, or even in terms of the more familiar phenomenological theories. It is particularly significant therefore that these results are well reprdoduced by the theory discussed above, as Erman $^{34}$ and Treloar $^{35}$ have shown. Thus, the theory accounts for the relationship of stress to strain in elastomers virtually throughout the range accessible to experimental measurement. ${ }^{35}$ The domains of constraint postulated by the theory exert their greatest effect at small strains. Inasmuch as the domains are distorted in proportion to the principal extension ratios $\lambda$, whereas the range of the phantom fluctuations is unaffected by deformation, the relative effect of the constraints in a given direction must vary inversely with the elongation $\lambda$. The effect vanishes as $\lambda$ is increased without limit. This description is over-simplified. It nevertheless explains qualitatively the attenuation of effects of the constraints at large strains or at high dilations. ${ }^{36}$ 


\section{P. J. FLORY}

The reduced nominal stress $\left[f_{\mathrm{ph}}^{*}\right]$ in the limit of high deformations or dilations emerges as the quantity that characterizes the molecular contribution of the network, as earlier experiments suggested. ${ }^{2,23}$ It is obviously essential to perform the extrapolation accurately, complications from curvature in plots of $\left[f^{*}\right] v s . \alpha^{-1}$ being avoided. Measurements on swollen networks offer the best procedure for this purpose.

As formally expressed, the theory takes account only of the covalent cross-linkages of the network embodied in the cycle rank $\xi$. Whether or not entanglements may increase the effective degree of interlinking is difficult to decide on purely theoretical grounds. It is an issue best resolved by experiments. If entanglements enhance the effective value of $\xi$, this enhancement should be reflected in $\left[f_{\mathrm{ph}}^{*}\right]$ determined by appropriate extrapolation of experimental measurements. The value thus determined may be compared with the "chemical" degree of interlinking, or the cycle rank.

Numerous experiments $\mathrm{s}^{29,32,40-45}$ show that $\left[f_{\mathrm{ph}}^{*}\right]$ obtained by extrapolation to $\alpha^{-1}=0$, or, in some instances $\left[f^{*}\right]$ measured at finite extensions, to be proportional to the chemical degree of interlinking. An intercept indicative of a threshold of "entanglement cross-linkages," often postualted, is not observed. The absolute magnitude of the chemical degree of interlinking is more difficult to establish with accuracy. In those instances where this objective has been achieved, the "elastic" and "chemical" degrees of interlinking are in good agreement. Included are networks of PDMS, ${ }^{29,32,40,42,43}$ poly(ethyl acrylate) $^{41}$ copoly(isoprene-styrene) ${ }^{44}$ and poly(cis-1,4-butadiene). ${ }^{45}$

Results deduced from the work of Mark and Sullivan $^{32}$ shown in Figure $7^{29}$ are illustrative of comparisons between limiting values of the reduced stress $\left[f_{\mathrm{ph}}^{*}\right]$ and degrees of interlinking. Values from elasticity measurements are somewhat higher than those obtained from the chemical structure, especially at low degrees of interlinking. Failure to attain ultimate elastic equilibrium may account for these departures. The results deduced from swelling equilibrium, which are not subject to this source of error, are in excellent agreement with the theoretical line based on the network structure as embodied in $\xi / V^{0}$.

The value of the parameter $\kappa$ appears to be related uniquely to the degree of interpenetration $\Gamma$

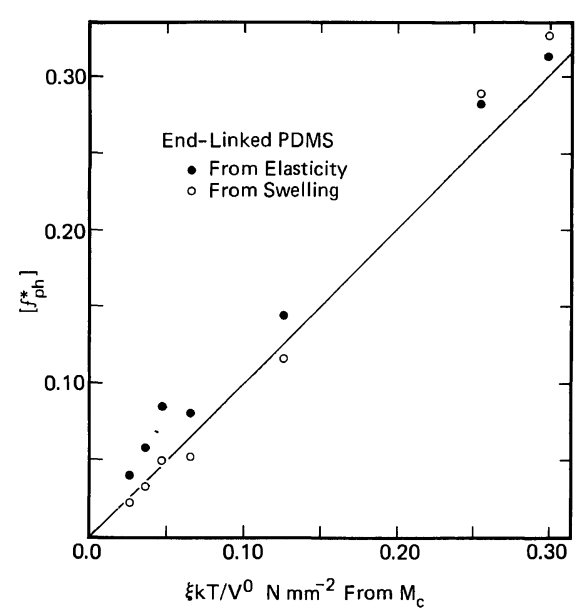

Figure 7. Limiting values of the reduced force $\left[f_{\mathrm{ph}}^{*}\right]$ for PDMS deduced from elasticity and swelling measurements of Mark and Sullivan ${ }^{32}$ plotted against $\xi k T / V^{0}$. From Erman and Flory. ${ }^{29}$

given by eq 7 ; i.e., ${ }^{29}$

$$
\kappa=\text { const } \Gamma=I\left\langle r^{2}\right\rangle_{0}^{3 / 2}\left(\mu_{\mathbf{J}} / V^{0}\right)
$$

where $I$ is an empirical parameter. Data available suggest that $I$ may be the same for all tetrafunctional networks. ${ }^{29}$ If this indication is verified, then it becomes possible to relate stress to strain on the basis of the degree of cross-linking which, in principle at least, is determinable from the chemical constitution of the network. Only the empirical parameter $\zeta$ would then be subject to arbitrary choice. Its role in refining agreement between theory and experiment is marginal.

\section{CONCLUSIONS}

The molecular theory here discussed provides a comprehensive account of rubber elasticity. It succeeds in relating the elastic equation of state to molecular constitution. This long sought objective is achieved with a latitude of choice in only one parameter. This parameter, $\kappa$, appears to be susceptible to independent determination, approximately at least, from the cycle rank $\xi$ that characterizes the connectivity of the network. Arbitrariness- in the choice of parameters may thus be reduced to an utter minimum.

The theory also accounts for the peculiar form of the dependence of the "elastic" contribution to the 
chemical potential of the diluent in a swollen network. $^{46}$ It provides the basis for a more exact treatment of strain birefringence in elastomeric networks. ${ }^{47.48}$ The theory appears to account also for the effects of functionality. ${ }^{49.50}$ These topics are beyond the scope of this review.

Acknowledgement. This work was supported by the Directorate of Chemical Sciences, Air Force Office of Scientific Research, Grant No. 82-0009.

\section{REFERENCES}

1. A. Ciferri, C. A. J. Hoeve, and P. J. Flory, J. Am. Chem. Soc., 83, 1015 (1961).

2. G. Allen, M. J. Kirkham, J. Padget, and C. Price, Trans. Faraday Soc., 67, 1278 (1971).

3. J. E. Mark, Rubber Chem. Technol., 46, 593 (1973).

4. P. J. Flory, Pure Appl. Chem., Macromol. Chem., 8, 1 (1972).

5. J. S. Higgins and R. S. Stein, J. Appl. Cryst., 11, 346 (1978).

6. P. J. Flory, Pure Appl. Chem., 56, 305 (1984).

7. P. J. Flory, Proc. R. Soc. London, Ser. A, 351, 351 (1976).

8. L. R. G. Treloar, "The Physics of Rubber Elasticity," 3rd ed, Oxford University Press, 1975.

9. E. Guth and H. M. James, Ind. Eng. Chem., 33, 624 (1941); H. M. James and E. Guth, J. Chem. Phys., 11, 455 (1943).

10. F. T. Wall, J. Chem. Phys., 11, 527 (1943).

11. L. R. G. Treloar, Trans. Faraday Soc., 39, 241 (1943).

12. P. J. Flory and J. Rehner, Jr., J. Chem. Phys., 11, 512 (1943).

13. W. Kuhn, J. Polym. Sci., 1, 380 (1946).

14. P. J. Flory, "Principles of Polymer Chemistry," Cornell Univ. Press, Ithaca, N. Y., 1953, Chapter XI.

15. H. M. James, J. Chem. Phys., 15, 651 (1947); H. M. James and E. Guth, ibid., 15, 669 (1947).

16. P. J. Flory and D. Y. Yoon, J. Chem. Phys., 61, 5358 (1974); P. J. Flory and V. W. C. Chang, Macromolecules, 9, 33 (1976).

17. P. J. Flory, Macromolecules, 15, 99 (1982).

18. P. J. Flory, Contemporary Topics in Polym. Sci., 2, 1 (1977)

19. P. J. Flory, Trans. Faraday Soc., 57, 829 (1961).

20. B. E. Eichinger, Macromolecules, 5, 496 (1972).

21. W. W. Graessley, Macromolecules, 8, 865 (1975).

22. P. J. Flory, A. Ciferri, and R. Chiang, J. Am. Chem. Soc., 83, 1023 (1961); R. Chiang, J. Phys. Chem., 70, $2348(1966)$.

23. G. Gee, Trans. Faraday Soc., 42, 585 (1946).

24. The restrictions on fluctuation here considered are operative at equilibrium. They are unrelated to the kinetic inhibitions that impede time-dependent re- laxation processes.

25. G. Ronca and G. Allegra, J. Chem. Phys., 63, 4990 (1975).

26. P. J. Flory, J. Chem. Phys., 66, 5720 (1977).

27. P. J. Flory, Polymer, 20, 1317 (1979).

28. P. J. Flory and B. Erman, Macromolecules, 15, 800 (1982).

29. B. Erman and P. J. Flory, Macromolecules, 15, 806 (1982).

30. H. Pak and P. J. Flory, J. Polym. Sci., Polym. Phys. $E d$., 17, 1845 (1979).

31. P. J. Flory and Y-I. Tatara, J. Polym. Sci., Polym. Phys. Ed., 13, 683 (1975).

32. J. E. Mark and J. L. Sullivan, J. Chem. Phys., 66, 1006 (1977).

33. See for example D. F. Jones and L. R. G. Treloar, $J$. Phys. D., 8, 1285 (1975).

34. B. Erman, J. Polym. Sci., Polym. Phys. Ed., 19, 829 (1981).

35. L. R. G. Treloar, Br. Polym. J., 121 (1982).

36. The theory does not apply, of course, to elastomers in which crystallization occurs as a result of deformation. ${ }^{37}$ The theory likewise does not anticipate the upturn in the tension which should eventually occur at high elongation, even in absence of crystallization, owing to approach of the chains to full extension. ${ }^{8.38}$ Ordinarily, observation of the predicted upturn is precluded by rupture of the sample. ${ }^{37}$ Exceptions occur when experimental conditions do not allow close approach to equilibrium ${ }^{38}$ or the distribution of chain lengths is bimodal. ${ }^{39}$

37. J. E. Mark, Polym. Eng. Sci., 19, 254, 409 (1979).

38. J. Furukawa, $Y$. Onouchi, S. Inagaki, and $\mathrm{H}$. Okamoto, Polym. Bull., 6, 381 (1981).

39. M. A. Llorente, A. L. Andrade, and J. E. Mark, Colloid Polym. Sci., 259, 1056 (1981); A. L. Andrade, M. A. Llorente, and J. E. Mark, J. Chem. Phys., 72, 2282 (1982); ibid., 73, 1439 (1982); J. E. Mark and M. Y. Tang, J. Polym. Sci., Polym. Phys. Ed., 22, 1849 (1984).

40. J. R. Falender, G. S. Y. Yeh, and J. E. Mark, $J$. Chem. Phys., 70, 5324 (1979).

41. B. Erman, W. Wagner, and P. J. Flory, Macromolecules, 13, 1554 (1980).

42. W. Oppermann and G. Rehage, Colloid Polym. Sci., 259, 1177 (1981).

43. P. J. Flory and B. Erman, J. Polym. Sci., Polym. Phys. Ed., 22, 49 (1984).

44. N. P. Ning, J. E. Mark, N. Iwamoto, and B. E. Eichinger, Macromolecules, 18, (1985), in press.

45. R. W. Brotzman and P. J. Flory, unpublished experiments on quantitatively cross-linked cis-1,4-polybutadiene.

46. R. W. Brotzman and B. E. Eichinger, Macromolecules, 14, 1445 (1981).

47. B. Erman and P. J. Flory, Macromolecules, 16, 1601 (1983). 


\section{P. J. FLORY}

48. B. Erman and P. J. Flory, Macromolecules, 16, 1607 (1983).

49. C.-Y. Jiang, L. Garrido, and J. E. Mark, J. Polym.
Sci., Polym. Phys. Ed., 22, 2281 (1984).

50. R. W. Brotzman, private communication. 